\title{
Autosomal dominant transmission of signal transduction and activator of transcription 1 (STAT1) mutation (Thr385Met) and extended lifespan
}

\author{
Kathryn Ruda Wessell*, Haig Tcheurekdjian, and Robert Hostoffer
}

\begin{abstract}
Introduction: Heterozygous mutations in signal transducer and activator of transcription 1 (STAT1) have been associated with selective deficiencies to mycobacterial or fungal infections. Recent reports revealed that patients found to carry de novo heterozygous mutations in STAT1 encoding specific amino acid substitutions can go on to develop progressive combined immunodeficiency, distinct from the limited susceptibilities to infection previously reported for heterozygous STAT1 mutations.

Objectives: We present a case of a mother and her son with chronic mucocutaneous candidiasis and T-cell dysfunction, both of whom lived longer than projected life expectancy with a heterozygous STAT1 mutation. The son lived to the age of 20 years and the mother to the age of 32 years.

Methods: The son's blood sequencing of STAT1 was performed on a pure T-cell lineage at The Hospital for Sick Children and the Canadian Center for Primary Immunodeficiency, Toronto, Ontario.

Results: STAT1 analysis revealed a heterozygous DNA binding domain mutation at Thr385Met. The patient proceeded to develop fulminant progressive multifocal leukoencephalopathy that ultimately led to his death. The patient's mother, although never confirmed to have STAT1 mutation with formal blood sequencing, had multiple comorbidities including progressive lymphopenia, hypogammaglobulinemia, recurrent Pseudomonas pneumonias with associated bronchiectasis, end-stage kidney disease requiring hemodialysis, and ultimately death due to multiple end organ failure associated sepsis.

Conclusion: This is the first autosomal dominant transmission of the STAT1 DNA binding domain Thr385Met mutation with an extended lifespan.

Statement of Novelty: Previous patients found to have heterozygous mutations of STAT1 that were associated with progressive combined immunodeficiency, arose de novo in each case. Presumed autosomal dominant transmission of a heterozygous STAT1 DNA binding domain Thr385Met mutation has not been reported.
\end{abstract}

\section{Introduction}

Signal transducer and activator of transcription (STAT1) is a critical signaling modulator downstream of a wide number of receptors. This transcription factor gets phosphorylated by Janus kinases upon ligation of these receptors (O'Shea et al. 2013). Following phosphorylation on tyrosine residues, STAT1 dimerizes and translocates to the nucleus to bind specific DNA sequences (Zakharova et al. 2003).

Mutations in STAT1 have been identified in a vast variety of clinical phenotypes (Dupuis et al. 2001, 2003; Chapgier et al. 2006a, 2006b, 2009; Boisson-Dupuis
Allergy Immunology Associates/University Hospitals Regional Hospitals Suite 110, 5915 Landerbrook Dr., Mayfield Heights, Ohio, United States 44124

*Corresponding author: Kathryn Ruda Wessell/kate.wessell@gmail.com
Submitted 3 October 2014

Accepted 14 November 2015

Available online 18 November 2015

LymphoSign Journal 3:13-17 (2016)

dx.doi.org/10.14785/lpsn-2015-0013 
et al. 2012; Sillevis Smitt and Kuijpers 2013). Recently heterozygous mutations in STAT1, mainly in the coiled-coil domain, were found to be associated with chronic mucocutaneous candidiasis (CMC) (Liu et al. 2011; van de Veerdonk et al. 2011; Nahum and Dalal 2014; Roifman 2014).

Mutations in the DNA binding domain have been identified in patients with progressive combined immunodeficiency (Sharfe et al. 2014). These patients suffer fatal virus infections usually before or during adolescence (Roifman 2014; Sharfe et al. 2014). The Th385Met mutation in the DNA binding domain has so far been reported as a de novo mutation in patients who die of viral infections early in life (Takezaki et al. 2012; Roifman 2014; Sharfe et al. 2014). We describe a case of heterozygous STAT1 mutation associated with autosomal dominant transmission, loss of lymphocyte function, autoimmune features, and diagnosis of severe infections and subsequent demise from progressive multifocal leukoencephalopathy (PML).

Progressive multifocal leukoencephalopathy is a demyelinating disease of the central nervous system caused by reactivation of John Cunningham virus (JCV) in a setting of cellular immunosuppression. Most often primary infection occurs asymptomatically in childhood, and the virus remains latent until reactivation. Traditionally, PML was observed in patients with advanced HIV infection and lymphoproliferative disorders and in transplant recipients. However, the widespread use of HIV antiretroviral drugs and the new selective immunomodulatory and immunosuppressive medications, such as Rituximab and Natalizumab, has modified the epidemiology (Lima 2013).

\section{Functional and clinical presentation}

A 19-year-old male presented with the previously established diagnosis of CMC. His previous medical history included failure to thrive from the age of 2 years, recurrent stomatitis, shingles, pneumonias, and bronchiectasis evident on chest computed tomography (CT). He was never followed consistently by a clinical immunologist at any point in his life. He was hospitalized for treatment of a pulmonary empyema and a cold immune-mediated hemolytic anemia resistant to many therapies, for which he subsequently received Rituximab prior to presentation. He was physically well at the visit.
In addition to the described medical history, he was also previously diagnosed with hypothyroidism, for which he received inconsistent medical therapy. His mother had been diagnosed with CMC and a T-cell dysfunction in early childhood. She had spent much of her early life hospitalized for intravenous anti-fungal therapy. She had evidence of bronchiectasis on chest CT. She had end-stage renal disease secondary to amyloidosis and hypothyroidism. She ultimately developed hypogammaglobulinemia in addition to her T-cell dysfunction as evidenced by abnormal mitogen-antigen testing. She died at the age 32 years of end organ failures and associated recurrent Pseudomonas and Aspergillus pneumonias and sepsis.

Upon presentation, his previous labs (Table 1) revealed low IgA, initial abnormal pneumococcal titers with normal vaccine response, and abnormal response to Candida and Tetanus lymphocyte antigen stimulation. His chest CT demonstrated clear bronchiectatic changes and mucus plugging.

Based on the patient's clinical history, diagnostic testing for STAT1 mutation was completed at the initial visit. His blood sample was sent to The Hospital for Sick Children and the Canadian Center for Primary Immunodeficiency in Toronto, Ontario. STAT1 analysis revealed a heterozygous DNA binding domain mutation at Thr385Met. (Figure 1) Primary Epstein-Barr virus cell lines were used for the mutation analysis of the STAT1 mutation discovered in this patient.

The following month, clear physical and neurological deterioration had progressed in the patient. He reported right-sided weakness for which he presented to an area hospital. Magnetic resonance imaging (MRI) of the brain was completed showing multiple foci of abnormal hyperintensity signals. He was admitted to the tertiary care hospital for further evaluation. This was based on the known STAT1 mutation and abnormal brain MRI in conjunction with progressing physical deterioration specifically on the right side of his face and arm, leg muscle weakness, and dysarthria-dysphagia. Positive JCV DNA was isolated and reported in the cerebral spinal fluid (CSF), and brain MRI was interpreted to be consistent with progressive multifocal leukoencephalopathy (PML).

The JCV continued to cause rapidly progressive neuromuscular deficits. He was transferred to the National Institutes of Health, Bethesda, Maryland, for initiation 
Table 1: Lab results of 20 -year-old male patient.

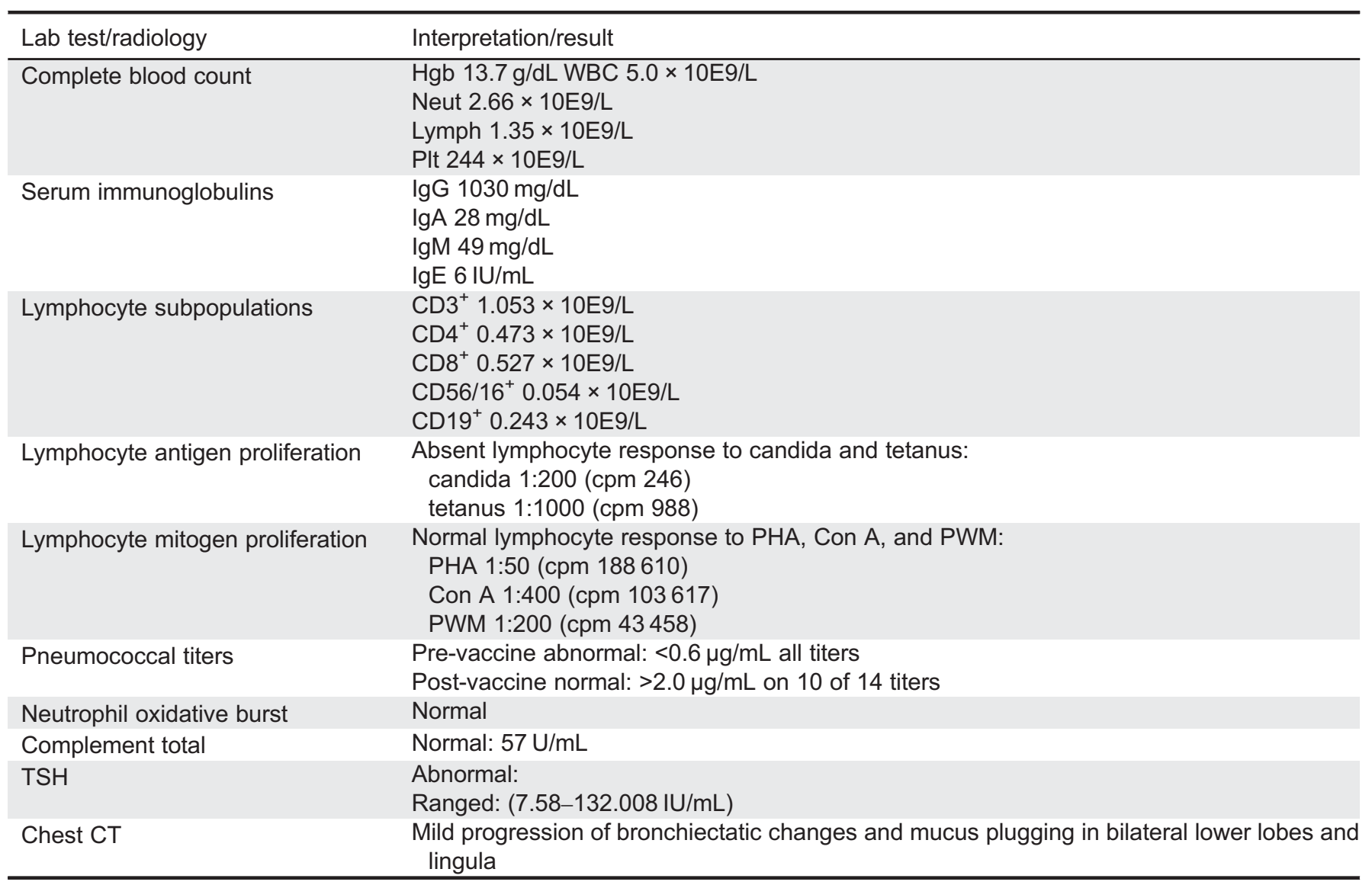

Note: cpm, counts per minute; PHA, phytohemagglutinin; Con A, concanavalin A; PWM, pokeweed mitogen.

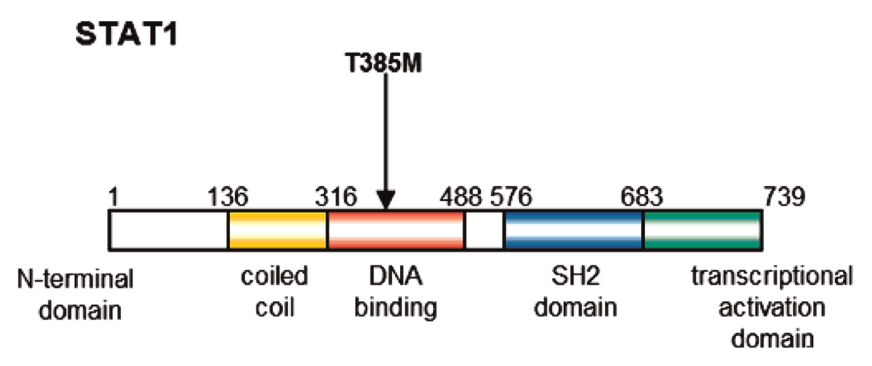

Figure 1: STAT 1 mutation loci (Sharfe et al. 2014).

of interferon alpha (IFN- $\alpha$ ) therapy. After a few weeks of IFN- $\alpha$ treatment 3 times per week, his viral load decreased in the CSF and his rate of neuromuscular progression declined. These gains were proven temporary, his deficits subsequently progressed, and he died during treatment.

This patient synopsis demonstrates an autosomal dominant transmission of heterozygous STAT1 Thr385Met mutation, opposed to the cases previously described (Sharfe et al. 2014) in which the varying STAT1 heterozygous mutations arose de novo. The patient's mother was the oldest living patient with this presumed specific STAT1 mutation, and our patient represented the oldest patient with this specific monoallelic mutation verified, living until 20 years of age. His development of PML suggests that this risk must be considered in the management of STAT1 mutation cases.

\section{Discussion}

Patients with mono-allelic mutations in the DNA binding domain appear to present with deteriorating cellular as well as humoral immunity, leading to fatal viral infections (Sharfe et al. 2014). In particular, the T385M mutation has been shown to be associated with catastrophic outcome during childhood or adolescence. It was therefore assumed that because of its severity this mutant can only appear de novo.

Similar to others carrying the same mutation, we showed that our patient suffered repeated infections and chronic bronchiectasis. He also had lower than normal lymphocyte counts as well as $\mathrm{CD}^{+} \mathrm{T}$ cells. 
Eventually he died of JCV encephalitis. This infection followed the use of Rituximab he received for treatment of hemolytic anemia. It is therefore possible that Ritaximab may have contributed to the susceptibility of JCV. Although this possibility cannot be excluded, another patient with a mutation in the DNA binding domain also died of JCV encephalitis without the use of immunosuppression, suggesting the combined immunodeficiency developed in these patients may be sufficient to predispose them to an overwhelming JCV infection (Sharfe et al. 2014).

In contrast with other patients with the T385M mutation, this patient survived until the age of 20 years. Moreover, his mother likely had the same mutation, and she survived until the age of 32 years. She was diagnosed in early childhood with chronic candidiasis and T-cell dysfunction, and she subsequently developed hypogammaglobulinemia.

These cases indicate that patients with T385M mutations may survive into adulthood and have children. This variability in clinical course should not be surprising as many other mono-allelic disorders appear widely diverse.

In conclusion, we reported on the first documented case of survival into adulthood of a patient with the T385M mutation in STAT1.

\section{Acknowledgement}

The authors thank Dr. Chaim Roifman for performing the genetic analysis of STAT1.

\section{REFERENCES}

Boisson-Dupuis, S., Kong, X.F., Okada, S., Cypowyj, S., Puel, A., Abel, L., and Casanova, J.L. 2012. Inborn errors of human STAT1: Allelic heterogeneity governs the diversity of immunological and infectious phenotypes. Curr. Opin. Immunol. 24:364-378. PMID: 22651901. doi: 10.1016/j.coi.2012.04.011.

Chapgier, A., Boisson-Dupuis, S., Jouanguy, E., Vogt, G., Feinberg, J., Prochnicka-Chalufour, A., Casrouge, A., Yang, K., Soudais, C., Fieschi, C., Santos, O.F., Bustamante, J., Picard, C., de Beaucoudrey, L., Emile, J.F., Arkwright, P.D., Schreiber, R.D., RolinckWerninghaus, C., Rösen-Wolff, A., Magdorf, K., Roesler, J., and Casanova, J.L. 2006a. Novel STAT1 alleles in otherwise healthy patient with mycobacterial disease. PLoS Genet. 2(8): e131. doi: 10.1371/journal. pgen.0020131.

Chapgier, A., Kong, X.F., Boisson-Dupuis, S., Jouanguy, E., Averbuch, D., Feinberg, J., Zhang, S.Y., Bustamante, J., Vogt, G., Lejeune, J., Mayola, E., de Beaucoudrey, L., Abel, L., Engelhard, D., and Casanova, J. 2009. A partial form of recessive STAT1 deficiency in humans. J. Clin. Invest. 119:1502-1514. PMID: 19436109. doi: 10.1172/JCI37083.

Chapgier, A., Wynn, R.F., Jouanguy, E., Filipe-Santos, O., Zhang, S., Feinberg, J., Hawkins, K., Casanova, J. L., and Arkwright, P.D. 2006b. Human complete Stat-1 deficiency is associated with defective type I and II IFN responses in vitro but immunity to some low virulence viruses in vivo. J. Immunol. 176:50785083. PMID: 16585605. doi: 10.4049/jimmunol. 176.8.5078.

Dupuis, S., Dargemont, C., Fieschi, C., Thomassin, N., Rosenzweig, S., Harris, J., Holland, S.M., Schreiber, R.D., and Casanova, J.L. 2001. Impairment of mycobacterial but not viral immunity by a germline human STAT1 mutation. Science. 293:300-303. PMID: 11452125. doi: 10.1126/science.1061154.

Dupuis, S., Jouanguy, E., Al-Hajjar, S., Fieschi, C., AlMohsen, I.Z., Al-Jumaah, S., Yang, K., Chapgier, A., Eidenschenk, C., Eid, P., Al Ghonaium, A., Tufenkeji, H., Frayha, H., Al-Gazlan, S., Al-Rayes, H., Schreiber, R.D., Gresser, I., and Casanova, J.L. 2003. Impaired response to interferon-alpha/beta and lethal viral disease in human STAT1 deficiency. Nat. Genet. 33:388-391. PMID: 12590259. doi: 10.1038/ng1097.

Lima, M. 2013. Progressive Multifocal Leukoenephalopathy: New Concepts. Arq. Neuropsiquiatr. 71(9B): 699-702. PMID: 24141508. doi: 10.1590/0004282X20130154.

Liu, L., Okada, S., Kong, X.F., Kreins, A.Y., Cypowyj, S., Abhyankar, A., Toubiana, J., Itan, Y., Audry, M., Nitschke, P., Masson, C., Toth, B., Flatot, J., Migaud, M., Chrabieh, M., Kochetkov, T., Bolze, A., Borghesi, A., Toulon, A., Hiller, J., Eyerich, S., Eyerich, K., Gulácsy, V., Chernyshova, L., Chernyshov, V., Bondarenko, A., Grimaldo, R.M., Blancas-Galicia, L., Beas, I.M., Roesler, J., Magdorf, K., Engelhard, D., Thumerelle, C., Burgel, P.R., Hoernes, M., Drexel, B., Seger, R., Kusuma, T., Jansson, A.F., Sawalle-Belohradsky, J., Belohradsky, B., Jouanguy, E., Bustamante, J., Bué, M., Karin, N., Wildbaum, G., Bodemer, C., Lortholary, O., Fischer, A., Blanche, S., Al-Muhsen, S., Reichenbach, J., Kobayashi, M., Rosales, F.E., Lozano, C.T., Kilic, S.S., Oleastro, M., Etzioni, A., Traidl-Hoffmann, C., Renner, E.D., Abel, L., Picard, C., Maródi, L., Boisson-Dupuis, S., Puel, A., and 
Casanova, J.L. 2011. Gain-of-function human STAT1 mutations impair IL-17 immunity and underlie chronic mucocutaneous candidiasis. J. Exp. Med. 208:1635-1648. PMID: 21727188. doi: 10.1084/ jem.20110958.

Nahum, A., and Dalal, I. 2014. Clinical manifestations associated with novel mutations in the coiled-cloi domain of STAT1. LymphoSign J. 1(2):97-103. doi: 10.14785/lpsn-2014-0021.

O'Shea, J.J., Holland, S.M., and Staudt, L.M. 2013. JAKs and STATs in immunity, immunodeficiency, and cancer. N. Engl. J. Med. 368(2):161-170. PMID: 23301733. doi: 10.1056/NEJMra1202117.

Roifman, C.M. 2014. Monoallelic STAT1 mutations and disease patterns. LymphoSign J. 1(2):57-59. doi: 10.14785/lpsn-2014-0011.

Sharfe, N., Nahum, A., Newell, A., Dadi, H., Ngan, B., Pereira, S.L., Herbrick, J.A., and Roifman, C. M. 2014. Fatal combined immunodeficiency associated with heterozygous mutation in STAT1. J. Allergy Clin. Immunol. 133(3):807-817. PMID: 24239102. doi: 10.1016/j.jaci.2013.09.032.

Sillevis Smitt, J.H., and Kuijpers, T.W. 2013. Cutaneous manifestations of primary immunodeficiency. Curr.
Opin. Pediatr. 25(4):492-497. PMID: 23744097. doi: 10.1097/MOP.0b013e3283623b9f.

Takezaki, S., Yamada, M., Kato, M., Park, M.J., Maruyama, K., Yamazaki, Y., Chida, N., Ohara, O., Kobayashi, I., and Ariga, T. 2012. Chronic mucocutaneous candidiasis caused by a gain-of-function mutation in the STAT1 DNA-binding domain. J. Immunol. 189:1521-1526. PMID: 22730530. doi: 10.4049/ jimmunol.1200926.

van de Veerdonk, F.L., Plantinga, T.S., Hoischen, A., Smeekens, S.P., Joosten, L.A.B., Gilissen, C., Arts, P., Rosentul, D.C., Carmichael, A.J., Smits-van der Graaf, C.A., Kullberg, B.J., van der Meer, J.W., Lilic, D., Veltman, J.A., and Netea, M.G. 2011. STAT1 mutations in autosomal dominant chronic mucocutaneous candidiasis. N. Engl. J. Med. 365:54-61. PMID: 21714643. doi: 10.1056/NEJMoa1100102.

Zakharova, N., Lymar, E.S., Yang, E., Malik, S., Zhang, J. J., Roeder, R.G., and Darnell, J.E. Jr.. 2003. Distinct transcriptional activation functions of STAT $1 \alpha$ and STAT1 $\beta$ on DNA and chromatin templates. J. Biol. Chem. 278:43067-43073. PMID: 12939262. doi: 10.1074/jbc.M308166200. 